\title{
New Results on X-ray Models and Atomic Data
}

\author{
Jelle S. Kaastra ${ }^{1}$, Rolf Mewe ${ }^{1}$, \& Ton Raassen ${ }^{1,2}$ \\ ${ }^{1}$ SRON, Sorbonnelaan 2, 3584 CA Utrecht, The Netherlands \\ ${ }^{2}$ Astronomical Institute Anton Pannekoek, Amsterdam, The Netherlands
}

\begin{abstract}
We discuss the most recent developments of the spectral analysis package SPEX. We report on the progress made in updating the atomic data that are used in the spectroscopic code. We also present a set of spectral models that are used for the analysis of high-resolution X-ray spectra of photo-ionized plasmas such as occur in active galactic nuclei. These models include absorption line spectroscopy of photoionized layers. The importance and diagnostic power of inner-shell transitions is shown. We illustrate our results with several examples of observed spectra obtained with the XMM-Newton and Chandra grating spectrometers.
\end{abstract}

\section{Introduction}

Starting in the early seventies (Mewe 1972) we have developed a spectral code for the X-ray emission of optically thin plasmas. Milestones in this development were the Mewe-Gronenschild code (Mewe et al. 1985, 1986), the mekal model (Mewe et al. 1995) and the start of the SPEX project (Kaastra et al. 1996). The mekal model is a model for the calculation of emission spectra from optically thin, collisionally ionized plasmas. It is included in the popular XSPEC package and in a more complete form (including thermal Doppler broadening and nonequilibrium ionization (NEI) variants) in our own SPEX package, version 1. The SPEX package contains apart from the software to produce these thermal emission spectra also modules to calculate other line/continuum emission or absorption models, hydrodynamical models for supernova remnants, differential emission measure (DEM) analysis tools, spectral fitting and plotting tools, and tools to display relevant physical information of the plasma, ions or transitions.

Already at the time of the release of the mekal code and the SPEX package it became clear that updates are needed for various reasons. The advent of highresolution X-ray spectroscopy through the launch of Chandra and XMM-Newton demanded for higher precision in wavelengths, a larger number of spectral lines to be included and more accurate atomic data. This also required more efficient use of computing power. Therefore we started updating our SPEX package. Version 2.0 is now available (see http://www.sron.nl/divisions/hea/spex/index.html). More background information on version 2.0 is given by Kaastra et al. (2002a).

We have made several updates of the mekal code that are available now in version 2.0 of SPEX. These include wavelength corrections according to Phillips et al. (1999), based upon Solar flare spectra; update of the line power of the 
strongest Fe XVII lines according to Doron \& Behar (2002), and several minor corrections.

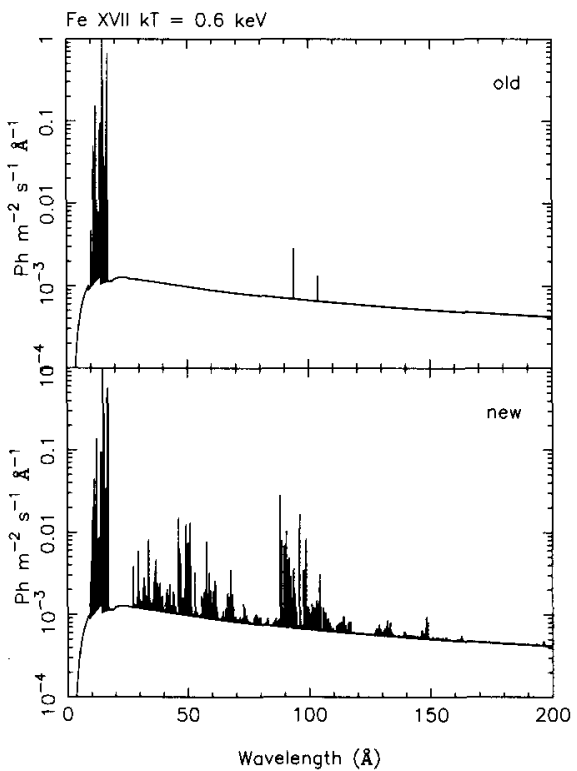

Figure 1. Fe XVII emission spectrum using mekal ("old") and our updated calculations ("new").

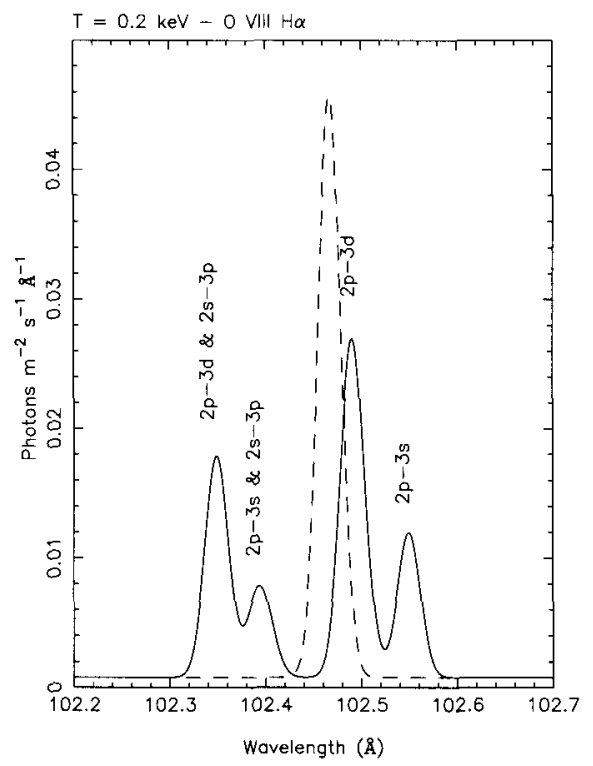

Figure 2. O VIII $\mathrm{H} \alpha$ emission spectrum using mekal (dashed line) and our updated calculations (solid line).

We are also working on a more ambitious project to improve and extend the atomic data for all transitions using different sources of data, such as calculations obtained using the HULLAC and FAC atomic codes and data available from the literature. For example, the mekal code has a subset of the strongest Fe XVII lines; the line powers used in that code were determined by scaling the old MeweGronenschild temperature-dependent line powers to the level as calculated with HULLAC at the temperature of maximum emissivity. In our new work, we make full use of the HULLAC calculations (provided by D. Liedahl) for this ion and the other L-shell ions of iron. This also allows us to include many more transitions, see Fig. 1 for an example. In particular in the soft X-ray and EUV band we have many more lines now. At the harder X-rays the differences are not very large, although the inclusion of higher principal quantum numbers in our updated calculations produces more flux in specific narrow wavelength bands (cf. Brickhouse et al. 2000).

Another example of our update is given in Fig. 2. The Mekal code contains for hydrogenic ions only the Lyman $\alpha, \beta, \gamma, \delta$ and $\epsilon$ lines, as well as $\mathrm{H} \alpha$. We used the FAC code of M.F. Gu to extend the calculations to include all transitions up to $n=10$. The figure shows the importance of the inclusion of the appropriate atomic data, since $H \alpha$ is now resolved into its 6 components.

Currently we are testing the newly included data and as soon as this has been finished they will be included in the latest SPEX version; we will announce 
this at the afore mentioned web page. Up to then, SPEX version 2.0 contains the updated mekal code.

We have also included absorption models for photo-ionized plasmas in version 2.0 of SPEX. For more details about these models we refer to Kaastra et al. (2002a). Examples of their application can be found in Kaastra et al. (2002b) and Steenbrugge et al. (2003).

Of particular interest in the application of these absorption models are the inner shell transitions. The importance of these lines in the context of absorption models has first been demonstrated by Sako et al. (2001) in their discovery of the Fe-M Unresolved Transition Array in a quasar spectrum. Since then, also the importance of $1 \mathrm{~s}-n \mathrm{p}$ inner shell transitions in oxygen ions has been shown. For instance, in the Seyfert 1 galaxy NGC 5548, XMM-Newton RGS observations showed the presence of O IV-O VIII absorption lines. In fact it can be shown that for low ionization parameters and unsaturated columns, these inner shell oxygen transitions are the strongest absorption features expected in the RGS and Chandra LETGS bands (mainly due to the large cosmic oxygen abundance). Unfortunately, different atomic codes predict different wavelengths for these transitions, with differences up to the resolution of the RGS and LETGS. Laboratory measurements of the wavelengths are urgently needed.

Acknowledgments. The Space Research Organisation of the Netherlands (SRON) is supported financially by NWO, the Netherlands Organisation for Scientific Research.

\section{References}

Brickhouse, N.S., Dupree, A.K., Edgar, R.J., et al. 2000, ApJ 530, 387

Doron, R., \& Behar, E. 2002, ApJ, 574, 518

Kaastra, J.S., Mewe, R., \& Nieuwenhuijzen, H. 1996, in UV and X-ray Spectroscopy of Astrophysical and Laboratory Plasmas, p. 411, eds. K. Yamashita \& T. Watanabe, Tokyo, Univ. Ac. Press.

Kaastra, J.S., Mewe, R., \& Raassen, A.J.J., 2002a, in New Visions of the X-ray Universe in the XMM-Newton and Chandra Era, ESA SP, in press

Kaastra, J.S., Steenbrugge, K.C., Raassen, A.J.J., et al. 2002b, A\&A 386, 427

Mewe, R., 1972, Solar Phys. 22, 459

Mewe, R., Gronenschild, E.H.B.M., \& van den Oord, G.H.J. 1985, A\&A Supp 62, 197

Mewe, R., Lemen, J.R., \& van den Oord, G.H.J. 1986, A\&A Supp 65, 511

Mewe, R., Kaastra, J.S., \& Liedahl, D.A. 1995, Legacy 6, 16

Phillips, K.J.H., Mewe, R., Harra-Murnion, L.K., et al. 1999, A\&A Supp 138, 381

Sako, M. et al. 2001, A\&A, 365, 168

Steenbrugge, K.C., Kaastra, J.S., de Vries, C.P., \& Edelson, R. 2003, A\&A 402, 477 